Research Paper:

\title{
Lipid Profile and Risk Factors of Cardiovascular Diseases in Adult Candidates for Lumbar Disc Degenerative Disease Surgery
}

Shiva Mashinchi ${ }^{1}$ (D), Zahra Hojjati-Zidashti2* ${ }^{2}$ (D), Shahrokh Yousefzadeh-Chabok ${ }^{3,4}$ (D)

1. MSc. in Sport Physiology, Department of Physical Education, Rasht Branch, Islamic Azad University, Rasht, Iran

2. Associate Professor, Department of Physical Education, Rasht Branch, Islamic Azad University, Rasht, Iran

3. Professor of Neurosurgery, Neuroscience Research Center, Guilan University of Medical Sciences, Rasht, Iran

4. Guilan Road Trauma Research Center, Guilan University of Medical Sciences, Rasht, Iran

\begin{tabular}{|l|l|}
$\begin{array}{l}\text { Use yourdevic to scan } \\
\text { and read thearticle online }\end{array}$ & $\begin{array}{l}\text { Crtation Mashinchi Sh, Hojjati-Zidashti Z, Yousefzadeh-Chabok Sh. Lipid Profile and Risk Factors of Cardiovascular Diseas- } \\
\text { es in Adult Candidates for Lumbar Disc Degenerative Disease Surgery. Iran J Neurosurg. 2018; 4(3):157-166. http://dx.doi. } \\
\text { org/10.32598/irjns.4.3.157 }\end{array}$ \\
doli http://dx.doi.org/10.32598/irjns.4.3.157
\end{tabular}

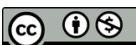

Article info:

Received: 29 November 2017

Accepted: 25 May 2018

Available Online: 01 July 2018

Keywords:

Lumbar disc degenerative diseases, Lipid profile, HighDensity Lipoprotein (HDL), Low-Density Lipoprotein (LDL), Triglyceride (TG), Risk factors for cardiovascular diseases

\section{ABSTRACT}

Background and Aim: There is no consensus over the relationship between lipid profiles and atherosclerosis risk factors with lumbar disc degeneration. This study aims to investigate the lipid profile and some risk factors of cardiovascular diseases in patients undergoing surgery for lumbar disc degeneration.

Methods and Materials/Patients: A correlational cross-sectional study was carried out on all candidates for lumbar disc degenerative diseases surgery at Poursina Hospital in Rasht City, Iran from July to December 2017 (130 adult patients aged 20-77 years). The obtained data were collected from the patients' medical file or asking them after obtaining written informed consent. The collected data were analyzed in SPSS V. 16 by performing the Chi-square and Fisher's exact tests to examine the relationship between the study variables. The significance level was set at $\mathrm{P} \leq 0.05$ for all tests.

Results: $60.8 \%$ of subjects had comorbid diseases, mostly hyperlipidemia, and hypertension. Their mean low-density lipoprotein and triglyceride levels were higher than normal and their mean total cholesterol was at borderline. Also, $74.6 \%$ and $84.6 \%$ of the patients had Body Mass Index and abdominal circumference higher than the normal range. Physical activity in $88.5 \%$ of subjects was below the desired level and $79.2 \%$ of the patients did not report a history of smoking. Inferential findings indicated a significant relationship between dyslipidemia and various types of lumbar disc degenerative diseases $(P<0.05)$.

Conclusion: The study showed dyslipidemia and some other risk factors of cardiovascular diseases, including obesity and inactivity in patients with lumbar disc degenerative disease. More research is needed to assess other atherosclerosis risk factors and types of lipids with respect to disc degenerative diseases.

\section{* Corresponding Author:}

Zahra Hojjati-Zidashti, PhD.

Address: Department of Physical Education, Rasht Branch, Islamic Azad University, Rasht, Iran

Tel: +98 (911) 1482499

E-mail:z_hoj@yahoo.com 


\section{Highlights}

- $60.8 \%$ of subjects had comorbid diseases.

- Hyperlipidemia and hypertension were the most reported comorbid diseases.

- The mean LDL and triglyceride levels were higher than normal and the mean total cholesterol was reported at borderline.

- $79.2 \%$ of patients did not report a history of smoking.

- Majority of the patients had BMI and abdominal circumference higher than normal and suboptimal physical activity.

\section{Plain Language Summary}

Lumbar Disc Degenerative diseases (LDD) are among the most important causes of lower back pain. Various environmental and genetic factors are involved in their development. In addition to physical activities, weight-induced pressure and abdominal obesity involved in lumbar disc degeneration, a direct relationship has been found between lipid profile and cardiovascular risk factors with disc degeneration and low back pain, especially in recent years. The current study was conducted with the aim of examining lipid profile, weight, BMI, smoking, physical activity and the history of some comorbid diseases as risk factors of cardiovascular diseases in patients undergoing surgery for lumbar disc degeneration. Based on the current study results, more than half of the subjects had comorbid diseases, mostly hyperlipidemia, and hypertension. The majority of subjects had BMI and abdominal circumference higher than normal and suboptimal physical activity but no report of smoking history. Inferential findings indicate a significant relationship between dyslipidemia and various types of lumbar disk degenerative diseases.

\section{Introduction}

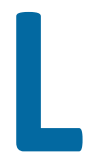

umbar Disc Degenerative diseases (LDD) are among the most important causes of low back pain, and various environmental and genetic factors are involved in their development [1-3]. The incidence and severity of degenerative Disc Diseases (DD) are associated with increasing age [4]. In the past, the heavy physical load was accounted for the most important cause of these diseases, while recent studies emphasize on a variety of factors $[1,5]$. In addition to physical and job activities, weight-induced pressure and abdominal obesity involved in lumbar disc degeneration [6, 7], a direct relationship between lipid profile and risk factors for atherosclerosis and Cardiovascular Diseases (CVD), with DD and Low Back Pain (LBP) has been shown in epidemiological and clinical studies, especially in recent years [7-9]. Importantly, the increasing prevalence of obesity, inactivity, and dyslipidemia -as the risk factors for CVD are currently considered $[9,10]$.

In Kauppila review study on atherosclerosis and DD/ LBP, the results of post-exposure studies have shown the relationship between lipid deposit lesions in the aorta and DD, as well as obstruction of the lumbar arteries and LBP. Clinical studies reveal the relationship between the calcification of aorta, LBP, and lumbar arteries stenosis with LBP and DD. Epidemiological studies have also suggested a stronger association between high levels of serum cholesterol, DD and LBP. Consequently, aortic atherosclerosis and stenosis of the spinal feeding arteries are associated with DD and LBP. According to this review study, cardiovascular risk factors have shown a weak relationship with DD and LBP, as it is only evident in cohort studies on the elderly or very large samples. More prospective clinical studies are required to further clarify the relationship between atherosclerosis and lower back disorders [9].

In an observational study by Hangai et al. on 270 adolescents aged 51-86 years participating in a health promotion program, the relationship between $\mathrm{DD}$ and Body Mass Index (BMI), Low-Density Lipoprotein (LDL), Triglyceride (TG), smoking, and some multiple factors associated with atherosclerosis were evaluated with a confidence interval of $95 \%$. The study results indicated the association of high BMI and high levels of LDL with DD which increases the likelihood of the association of cardiovascular risk factors with lumbar disc degenera- 
tion [7]. In a case-control study by Longo et al, regarding the serum lipid and disc herniation levels, two groups were included: A. 169 patients undergoing surgery for symptomatic disc herniation; and B. 169 patients undergoing a meniscus tear surgery. Their study showed significant higher concentrations of triglycerides and cholesterol in group A patients [8].

One of the main causes of disc degeneration seems to be poor nutritional supply to disc cells. Providing nutrients and disposing waste for large discs relies on nearby blood vessels. Disc central cells in an adult are approximately $8 \mathrm{~mm}$ away from the nearest blood supply [11, 12 ]. High levels of cholesterol and serum triglyceride as the risk factors for atherosclerosis are responsible for a decrease in the blood supply to the already poor vascularized intervertebral discs. Inadequate nutrition contributes to DD and it shows the relationship between the intervertebral disc diseases and the increased risk of dying from ischemic heart diseases [8]. In addition, several metabolic diseases can lead to disc degeneration through interfering with the normal biochemistry of the matrix structure, or adding new materials to the disc. For example, the patients with diabetes mellitus (a risk factor in the cardiovascular diseases) experience a significant decrease in hexosamine content of the disc core, an increase in hydroxyproline, and an enhancement in the activity of enzymes involved in the metabolism of carbohydrates. Moreover, disc proteoglycan synthesis changes through the varied sulfate content [11].

Obesity and overweight, in addition to the direct effect of increased load on lumbar spine [13, 14], and the role of biochemical and metabolic changes induced by lipids in the development of disc degeneration [10], are known to be effective in the incidence of lumbar vertebral diseases, as risk factors for vascular diseases such as elevated cholesterol, atherosclerosis, diabetes, and metabolic syndrome due to reduction of nutrients transported into the disc. It has also been introduced as a factor in decreasing physical activity indirectly [8, 15].

Sport activities have always been known as a significant factor in the prevention of chronic diseases, especially cardiovascular diseases [16]. Furthermore, lack of proper physical activity plays an important role in development of DD [15]; weight loss due to exercise, improvement of blood flow, lipid profile, and some blood biochemical factors along with other well-known mechanisms are the main reasons in this regard $[8,10]$.

Smoking habit also affects the incidence of LDD and increases the risk of hospitalization in affected patients [1,
17]. Obesity and tobacco are considered as potential risk factors for degenerative intervertebral disc diseases [8]. Kauppila review article (2009) reports a strong association between DD and LBP with smoking [9]. In the study by Jones et al. on 282 individuals older than 60, smoking at the time of examination had an effect on the development and severity of spinal osteophytes, with intermediate values for past smokers [1]. In the Finnish cohort study by Takatalo et al. on 558 young people with the mean age of 21 years, continuous smoking for at least 4 years was associated with DD among males [17].

In a part of a cohort study on 263529 Swedish construction workers participating in the National Program on Occupational Safety and Health from 1971 to 1992, smoking along with other factors such as overweight, was known to be effective in hospitalization [18]. Cigarette smoking, as shown in the laboratory studies on nucleolus cells isolated from cows, directly inhibits the proliferation of the disc cells and the synthesis of the extracellular matrix through nicotine. The result of the experiment on laboratory rats which were previously affected by smoking also indicates changes in collagen genes, and tissue changes leading to degeneration [11]. Smoking as one of the negative factors affecting the lipid profile, especially lowering HDL cholesterol, is also known to be a risk factor of cardiovascular diseases [4].

Given the high prevalence and cost, disability and absence from work due to lumbar diseases [19], the prevalence of low back pain among children and adolescents is increasing as well as adults [20]. Because there is not a suitable animal model due to significant anatomical differences between humans and laboratory animals [12] and some diagnostic methods used in human samples are expensive and dangerous [19], also considering the inadequate clinical studies and the inconsistency of their results $[1,21]$, carrying out clinical studies on available samples seems to be essential.

The current study was conducted with the aim of examining lipid profile, weight, BMI, smoking, physical activity and the history of some comorbid diseases as risk factors of cardiovascular diseases in patients undergoing surgery for lumbar disc degeneration.

\section{Methods \& Materials/Patients}

The present study was a correlational cross-sectional study conducted on all adult patients (130 patients aged 20-77 years) who were admitted to Poursina teaching hospital in Rasht City, Iran from July to December 2017, in order to undergo a surgery because of their LDD. The 
Table 1. The normal range of lipid profile with the tested kit

\begin{tabular}{ccc}
\hline Biochemistry Test & Unit & Normal Range \\
\hline Cholesterol & $\mathrm{mg} / \mathrm{dl}$ & $<200$ \\
TG & $\mathrm{mg} / \mathrm{dl}$ & $\mathrm{M}: 40-160 \mathrm{~F}: 35-135$ \\
HDL & $\mathrm{mg} / \mathrm{dl}$ & $\mathrm{M}: 35-50 \mathrm{~F}: 45-60$ \\
LDL & $\mathrm{mg} / \mathrm{dl}$ & $\begin{array}{c}\text { Desirable risk }<100 \text { medium risk } 130-160 \\
\text { High risk }>160\end{array}$ \\
\hline
\end{tabular}

ethical code of the study is IR.IAU.RASHT.REC.1395.50 and the initial stages of receiving the clinical trial code, have also been taken.

In order to carry out the study, after receiving the approval for the clinical research from the Poursina Hospital, the study objectives were described for the patients. After obtaining their written consent, their personal data and information about their current diseases, comorbidities and smoking history were extracted from their medical records, and the data deficiencies were completed through the face-to-face questionnaires (without the patient attendance if necessary).

In this study, in addition to subjects' lipid profile, other factors such as weight, BMI, smoking habit, physical activity level, history of comorbid diseases, including diabetes, high blood pressure, abnormal blood lipid, and history of cardiovascular diseases as other cardiovascular risk factors were investigated.

In order to investigate the patients' lipid profile, the test request was registered in the patients' medical records by the center's nutritionist and diet therapist, informing the relevant physician. The blood samples were taken after the patients' fasting for 12 hours, then the results of blood tests were evaluated by the laboratory of the hospital. The kits used in the laboratory were French Bionik laboratory kits, including Direct HDL and LDL kits, Enzymatic Cholesterol kit (CHOD-POD), and Triglyceride kit (GPO-POD) with a sensitivity of $1 \mathrm{mg} / \mathrm{dL}$. The normal ranges for lipid profile based on the above kits are presented in Table 1.

The patients' abdominal circumference, height, and weight were measured by the researcher, with a tape measure and the German Beurer digital scale. The BMI was calculated by dividing of weight in kilograms over height in meters squared. The individuals' activity level was measured by the Rapid Assessment of Physical Activity (RAPA) standard questionnaire. The findings were analyzed in SPSS 16 by performing the Chi-square and
Fisher's exact tests to examine the relationship between qualitative and ranking variables. The significance level was set at $P \leq 0.05$.

\section{Results}

$73 \%$ of subjects had experienced the symptoms of disc degenerative disease for more than one year, and $61 \%$ were hospitalized with the diagnosis of disc herniation. The Mean \pm SD age of the patients was $48.17 \pm 12.28$ years, with no significant differences between males and females. The number of men was 1.8 times the women.

\section{Lipid profile}

In the study of fasting lipid profile of the patients, $87.7 \%$ had dyslipidemia (at least one of the indicators was abnormal). The levels of total cholesterol, LDL, and triglyceride were more than normal in $46.2 \%, 68.5 \%$, and $46.2 \%$ of the subjects, respectively, and the level of HDL-cholesterol was less than normal in $44.6 \%$ of the patients. Among the components of patients' lipid profile, the Mean $\pm S D$ LDL-cholesterol with the level of $127.4 \pm 44.63 \mathrm{mg} / \mathrm{dL}$, and triglycerides with $16.73 \pm 7.17$ $\mathrm{mg} / \mathrm{dL}$ were reported as more than normal, and the Mean \pm SD total cholesterol level of $99.6 \pm 51.1 \mathrm{mg} / \mathrm{dL}$ was at the top of normal range (Table 2).

Blood serums of 4 patients were reported latescent by the laboratory because of the high level of triglyceride (more than $400 \mathrm{mg} / \mathrm{dL}$ ), and the highest reported triglyceride level was $949 \mathrm{mg} / \mathrm{dL}$ (about 6 times than the normal maximum in males).

Based on the inferential findings of this study, different components of lipid profile did not show any significant relationship with three groups of lumbar disc degenerations (disc herniation, spondylolisthesis, and spinal stenosis) in adult candidates for surgery. In this regard, there was no statistically significant relationship between total cholesterol ( $P=0.143), \mathrm{LDL}(P=0.386), \mathrm{HDL}$ $(P=0.73)$, and triglyceride $(P=0.205)$ with different kinds 
Table 2. Some indicators of lipid profile

\begin{tabular}{|c|c|c|c|}
\hline Variable & Condition & No. & $\%$ \\
\hline & Above normal & 60 & 46.2 \\
\hline \multirow[t]{2}{*}{ Total cholesterol } & Normal & 70 & 53.8 \\
\hline & Less than normal & 0 & 0 \\
\hline \multicolumn{2}{|c|}{ Total cholesterol, mg/dL (Mean \pm SD) } & \multicolumn{2}{|c|}{$199.6 \pm 51.1$} \\
\hline \multirow{3}{*}{ LDL } & Above normal & 89 & 68.5 \\
\hline & Normal & 41 & 31.5 \\
\hline & Less than normal & 0 & 0 \\
\hline \multicolumn{2}{|c|}{ LDL, mg/dL (Mean $\pm S D)$} & \multicolumn{2}{|c|}{$127.4 \pm 44.63$} \\
\hline \multirow{3}{*}{$\mathrm{HDL}$} & Above normal & 23 & 17.7 \\
\hline & Normal & 49 & 37.7 \\
\hline & Less than normal & 58 & 44.6 \\
\hline \multicolumn{2}{|c|}{$\mathrm{HDL}, \mathrm{mg} / \mathrm{dL}$ (Mean $\pm \mathrm{SD}$ ) } & \multicolumn{2}{|c|}{$44.4 \pm 13.89$} \\
\hline \multirow{3}{*}{ Triglyceride } & Above normal & 60 & 46.2 \\
\hline & Normal & 69 & 53.1 \\
\hline & Less than normal & 1 & 0.8 \\
\hline \multicolumn{2}{|c|}{ Triglyceride, mg/dL (Mean \pm SD) } & \multicolumn{2}{|c|}{$162.3 \pm 110.7$} \\
\hline \multirow{3}{*}{ Lipid profile } & Normal & 16 & 12.3 \\
\hline & & & \\
\hline & Abnormal & 114 & 87.7 \\
\hline
\end{tabular}

of lumbar degenerative diseases. However, this association was significant in the presence of dyslipidemia (at least one abnormal lipid indicator) (Table 3).

According to the Chi-square test results, there is a statistically significant relationship between the presence of dyslipidemia in three groups of patients suffer- ing from various types of lumbar degenerative diseases $(\mathrm{P}=0.018)$ (Figure 1).

Other cardiovascular risk factors

$74.6 \%$ of the subjects had a BMI greater than 25 , and $37.7 \%$ more than 30 . The Mean \pm SD subjects' BMI was

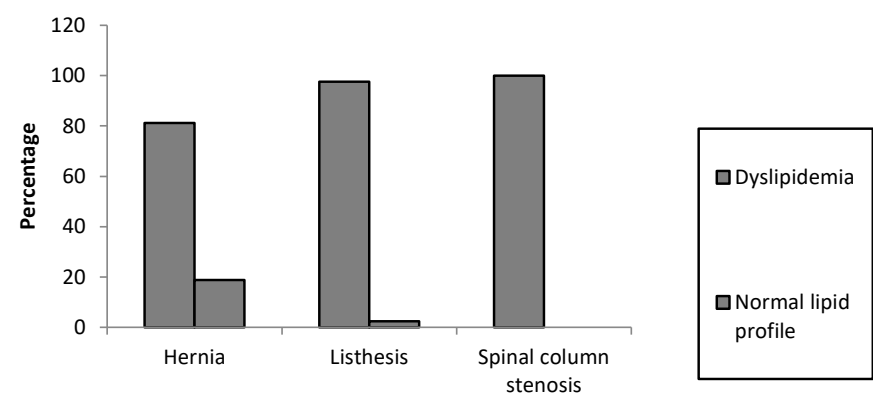

Figure 1. Distribution of dyslipidemia in three groups of patients suffering from various types of lumbar degenerative diseases 
Table 3. The inferential findings on the relationship between lipid profile and various Lumbar Disc Degenerative diseases

\begin{tabular}{|c|c|c|c|c|c|c|c|}
\hline \multirow{2}{*}{$\begin{array}{c}\text { Lipid Profile } \\
\text { Type }\end{array}$} & \multirow[b]{2}{*}{ Range } & \multicolumn{4}{|c|}{ Type of Disease } & \multirow[b]{2}{*}{ Test Type } & \multirow[b]{2}{*}{$\mathbf{P}$} \\
\hline & & Hernia & Listhesis & $\begin{array}{c}\text { Spinal Column } \\
\text { Stenosis }\end{array}$ & Total & & \\
\hline \multirow{2}{*}{$\begin{array}{l}\text { Total cholesterol } \\
(\mathrm{mg} / \mathrm{dL})\end{array}$} & Normal (\%) & 56.2 & 56.1 & 22.2 & 53.8 & \multirow{2}{*}{ Chi-square test } & \multirow{2}{*}{0.143} \\
\hline & >Normal (\%) & 43.8 & 43.9 & 77.8 & 46.2 & & \\
\hline \multirow{3}{*}{ LDL (mg/dL) } & Normal (\%) & 32.5 & 34.1 & 11.1 & 31.5 & \multirow{3}{*}{ Chi-square test } & \multirow{3}{*}{0.386} \\
\hline & & & & & & & \\
\hline & >Normal (\%) & 67.5 & 65.9 & 88.9 & 68.5 & & \\
\hline \multirow{2}{*}{$\mathrm{HDL}(\mathrm{mg} / \mathrm{dL})$} & Normal (\%) & 57.5 & 53.7 & 44.4 & 55.4 & \multirow{2}{*}{$\begin{array}{c}\text { Fisher's exact } \\
\text { test }\end{array}$} & \multirow{2}{*}{0.73} \\
\hline & $<$ Normal (\%) & 42.5 & 46.3 & 55.6 & 44.6 & & \\
\hline \multirow{3}{*}{$\begin{array}{l}\text { Triglyceride } \\
\text { (mg/dL) }\end{array}$} & Normal (\%) & 60 & 43.9 & 44.4 & 53.8 & \multirow{3}{*}{$\begin{array}{c}\text { Fisher's exact } \\
\text { test }\end{array}$} & \multirow{3}{*}{0.205} \\
\hline & & & & & & & \\
\hline & >Normal (\%) & 40 & 56.1 & 55.6 & 46.2 & & \\
\hline \multirow{3}{*}{ Lipid profile } & Normal (\%) & 18.8 & 2.4 & 0 & 12.3 & \multirow{3}{*}{ Chi-square test } & \multirow{3}{*}{0.018} \\
\hline & & & & & & & \\
\hline & Abnormal (\%) & 81.2 & 97.6 & 100 & 87.7 & & \\
\hline
\end{tabular}

$28.41 \pm 4.58 \mathrm{~kg} / \mathrm{m}^{2}$. Abnormal abdominal circumference was seen in $89.3 \%$ of women and $76.1 \%$ of men, and Mean $\pm S D$ abdominal circumference in the subjects was $104.73 \pm 16.57 \mathrm{~cm}$. In this study, the difference between mean abdominal circumference in men and women was statistically significant $(P=0.011)$.

According to the RAPA standard questionnaire score, $88.5 \%$ of the subjects had a suboptimal physical activity, and only $11.5 \%$ had an optimal physical activity based on their self-report. Also, 79.2\% of the subjects did not report any history of smoking in the past or present (Table 4).

A total of 79 subjects $(60.8 \%)$ had comorbid diseases (Table 5). A total of 107 comorbid diseases was observed in the subjects that high blood lipids (32.7\%) and hypertension (30.8\%) were the most common diseases. Inferential findings in the present study indicated a significant relationship between $\mathrm{BMI}$ and different types

Table 4. The prevalence of some cardiovascular risk factors in LDD patients

\begin{tabular}{ccc}
\hline \multicolumn{1}{c}{ Variable } & Status & $\%$ \\
\hline Body Mass Index & Higher than normal & 74.6 \\
\hline Abdominal circumference & Normal & 25.4 \\
& Higher than normal & 84.6 \\
Physical activity & Normal & 15.4 \\
& Suboptimal activity & 88.5 \\
Smoking & Optimal activity & 11.5 \\
& Having a history of smoking & 20.8 \\
\hline & Not having a history of smoking & 79.2 \\
\hline
\end{tabular}


Table 5. Frequency of different types of comorbid diseases

\begin{tabular}{ccc}
\hline Type of Comorbid Disease & No. & \% \\
\hline Diabetes & 23 & 21.5 \\
\hline High blood lipids & 35 & 32.7 \\
\hline High blood pressure & 33 & 30.8 \\
\hline Cardiovascular diseases & 16 & 15 \\
\hline Total & 107 & 100 \\
\hline & &
\end{tabular}

of degenerative disc diseases $(P<0.05)$, which was not significant for other variables $(P<0.05)$.

\section{Discussion}

In the study of fasting lipid profile of patients, a high percentage of them had dyslipidemia. The mean LDL and triglyceride levels in patients were higher than normal, and mean total cholesterol was at the top of normal range. The majority of the patients had BMI and abdominal circumference above normal and reported suboptimal physical activity; although smoking was not reported in most of them. Many epidemiological and clinical studies have shown a direct relationship between lipid profile, risk factors for atherosclerosis, and cardiovascular diseases with DD and LBP $[9,22]$. Dyslipidemia, comorbid diseases, and obesity, as risk factors for cardiovascular diseases, were also observed in our study patients.

Lipid profile

Kauppila review article (2009), has shown an association between lumbar arteries obstruction and stenosis as spinal feeding arteries, and aortic atherosclerosis with DD and LBP, and suggests a strong association between high serum cholesterol levels with DD and LBP. In Kauppila's study, cardiovascular risk factors have weaker associations with DD and LBP, as this association has only been seen in cohorts on the elderly or in large study samples [9].

Given that none of the factors of aortic atherosclerosis and lumbar arteries stenosis have been investigated in this study, it is not feasible to draw a conclusion in this regard, and the presence of dyslipidemia in this study as one of the risk factors for cardiovascular diseases is consistent with the results of the review article by Kauppila. In addition, the mean serum cholesterol levels in patients in this study were at the highest level and this is in line with the result of the study by Kauppila in this regard.

The results of Hangay et al. observational study on 270 adults indicate a relationship between high BMI value and high levels of LDL as the risk factors for cardiovascular diseases with Lumbar Disc Degeneration (LDD) [7]. It is also noteworthy that the risk of cardiovascular diseases is mentioned more associated with the serum LDL level than the total blood cholesterol according to some sources [23].

Longo et al. study showed a relationship between serum lipid levels and symptomatic disc herniation on two groups of patients; A. Patients undergoing surgery for disc herniation; and B. Patients undergoing surgery for meniscal tear. Group A showed higher triglyceride and total cholesterol level than group $B$ [8].

In the present study, the mean triglyceride level was reported above the normal, and total cholesterol level was at the top of normal range. As noted, insufficient nutrient supply to intervertebral discs, due to poor blood supply resulted from high cholesterol and triglyceride serum levels (known as risk factors of atherosclerosis) has been considered a predisposing factor for DD [8].

On the other hand, HDL as a good blood lipid helps eliminate bad cholesterol and prevent its accumulation in the arteries. This type of lipoprotein removes LDL from the blood and vascular walls, transports it to the liver, preserves the blood vessels safely and protects the heart. It is also used as an indicator to predict the risk factors for cardiovascular diseases [16, 23, 24].

\section{Other cardiovascular factors}

The high values of BMI and abdominal circumference in our patients are in line with the results of some previ- 
ous studies, including the studies by Takatalo et al. [17], Samartzis et al. [25], and Hangai et al. [7], about overweight and abdominal circumference in LDD patients. On the other hand, it disagrees with the results of some other studies like Jones et al. [1], Vidman et al. [21], and Daneshjoo and colleagues [26].

In addition to loading mechanical pressure on lumbar vertebra $[13,14]$, overweight and obesity are effective in degenerative disc diseases, through biochemical changes [10], effects on lipid profile, vascular and metabolic diseases $[8,10]$, and other known mechanisms. The results of the present study on the lack of physical activity in the subjects under study are in line with the results of the studies by Hengei et al. [7], Daneshjoo and colleagues [26], and disagrees with the results of the studies by Stephen et al. [27] and Takatalo et al. [17] about sport exercises and DD. The results of some studies suggest that regular and continuous physical activity reduces the risk of cardiovascular diseases more effectively compared to sport exercise $[8,27]$.

Regarding smoking as another risk factor for cardiovascular diseases, the results of the present study is inconsistent with some of the previous ones, including the studies by Kauppila [9], Takatalo et al. [17], and Jones et al. [1] which all referred to smoking in patients with degenerative disc diseases. It seems that the difference between the range of the subject's age in the present and previous studies and the lower number of samples could have had an impact on the results of this research. In addition, smoking seems to be more prevalent in the community, and patients' lack of integrity in reporting can be another reason for this discrepancy in results.

In Wahlstorm et al. study, the smoking factor along with other factors such as weight is known to be effective in hospitalization of the patients [18]. The results of the present study are also inconsistent with the result of Wahlstrom study, because in addition to the above mentioned cases, the majority of participants in the present study, in contrast to the construction workers participating in the study by Wahlstorm et al. were women, and smoking in women is significantly less than men, especially in the Iranian community.

In a demographic study conducted by Stephen Pye et al. to investigate the effects of lifestyle factors on DD, no significant effect of smoking was observed on peoples' LDD radiographic features [27]. A study by Liuke et al. on 129 middle-aged men showed no significant relationship between smoking and the prevalence of DD [6].
The result of the present study is consistent with the results of the two previously mentioned studies.

As it was mentioned, smoking in addition to its direct effect on the proliferation of disc cells and the synthesis of extracellular matrix [11] is effective in lumbar disc degeneration, through a negative effect on lipid profile and a decrease in HDL cholesterol [24].

This study had some limitations. First, failure to record the PMH (Past Medical History) in patient's medical records resulting from available medical documentation and the relatives of the patients or their attendants, do not necessarily mean that they had no previous diseases. Also, recent controlled lipid profile of some patients, due to drug use, does not necessarily indicate the lack of lipid profile's effect on the incidence and severity of the disease in the long term in the past.

Based on our study results, we suggest that the lipid profile of the patients with lumbar disc degeneration diseases should be regularly examined and controlled. Moreover, in order to prevent lumbar disc degeneration, special attention should be paid to the weight control, abdominal obesity, blood lipid and pressure as risk factors of cardiovascular diseases. Also, doing regular exercises and quitting smoking are recommended.

It is also suggested that a similar study be carried out with a larger sample size with more different study populations. Another study can be conducted to monitor the patients' lipid profile for a long time in order to investigate its association with the lumbar disc degeneration. Considering the significant relationship observed between dyslipidemia and the type of lumbar degenerative diseases and the role of various types of blood lipids in lumbar degenerative diseases, further research is recommended.

\section{Conclusion}

The study showed dyslipidemia and some other risk factors of cardiovascular diseases including obesity and inactivity in LDD patients. More research is needed to assess other atherosclerosis risk factors and types of lipids.

\section{Ethical Considerations}

\section{Compliance with ethical guidelines}

This study was approved by the Ethics Committee of Islamic Azad University, Rasht Branch (Code: IR.IAU. RASHT.REC.1395.50). 
Funding

The present paper was extracted from the MSc. thesis of the first author, in Department of Physical Education, Rasht Branch, Islamic Azad University.

\section{Authors' contributions}

All authors contributed in designing, running, and writing all parts of the research.

\section{Conflict of interest}

\section{The authors declared no conflict of interest.}

\section{Acknowledgments}

We sincerely thank Dr. Mesbah Dibavand and Dr. Ahmad Kheiri (the honorable neurosurgery residents), Ms Parvaneh Roodpeima and Ms Sabora Rahmati (the supervisors of the Department of Neurosurgery at Poursina teaching hospital in Rasht), and all the nurses and medical staff who helped us during this study. We would also express our special thanks to Poursina Clinical Research Development Unit, Guilan University of Medical Sciences, Rasht City, Iran.

\section{References}

[1] Jones G, White C, Sambrook P, Eisman J. Allelic variation in the vitamin $D$ receptor, lifestyle factors and lumbar spinal degenerative disease. Annals of the Rheumatic Diseases. 1998; 57(2):94-9. [DOI:10.1136/ard.57.2.94] [PMID] [PMCID]

[2] Livshits G, Popham M, Malkin I, Sambrook PN, MacGregor $\mathrm{AJ}$, Spector T, et al. Lumbar disc degeneration and genetic factors are the main risk factors for low back pain in women: The UK Twin Spine study. Annals of the Rheumatic Diseases. 2011; 70(10):1740-5. [DOI: 10.1136/ard.2010.137836] [PMID] [PMCID]

[3] Luoma K, Riihimäki H, Luukkonen R, Raininko R, Viikari-Juntura E, Lamminen A. Low back pain in relation to lumbar disc degeneration. Spine. 2000; 25(4):487-92 [DOI:10.1097/00007632-200002150-00016] [PMID]

[4] Teraguchi M, Yoshimura N, Hashizume H, Muraki S, Yamada H, Minamide A, et al. Prevalence and distribution of intervertebral disc degeneration over the entire spine in a population-based cohort: The Wakayama Spine study. Osteoarthritis and Cartilage. 2014; 22(1):104-10. [DOI:10.1016/j. joca.2013.10.019] [PMID]

[5] Liuke M, Solovieva S, Lamminen A, Luoma K, Leino-Arjas $\mathrm{P}$, Luukkonen R, et al. Disc degeneration of the lumbar spine in relation to overweight. International Journal of Obesity. 2005; 29(8):903-8. [DOI:10.1038/sj.ijo.0802974] [PMID]
[6] Battié MC, Videman T, Levälahti E, Gill K, Kaprio J. Genetic and environmental effects on disc degeneration by phenotype and spinal level: A multivariate twin study. Spine. 2008; 33(25):2801-8. [DOI:10.1097/BRS.0b013e31818043b7] [PMID]

[7] Hangai M, Kaneoka K, Kuno S, Hinotsu S, Sakane M, Mamizuka N, et al. Factors associated with lumbar intervertebral disc degeneration in the elderly. The Spine Journal. 2008 8(5):732-40. [DOI:10.1016/j.spinee.2007.07.392] [PMID]

[8] Longo UG, Denaro L, Spiezia F, Forriol F, Maffulli N, Denaro V. Symptomatic disc herniation and serum lipid levels. European Spine Journal. 2011; 20(10):1658-62. [DOI:10.1007/ s00586-011-1737-2] [PMID] [PMCID]

[9] Kauppila L. Atherosclerosis and disc degeneration/lowback pain: A systematic review. European Journal of Vascular and Endovascular Surgery. 2009; 37(6):661-70. [DOI:10.1016/j ejvs.2009.02.006] [PMID]

[10] Samartzis D, Karppinen J, Chan D, Luk KD, Cheung KM. The association of lumbar intervertebral disc degeneration on magnetic resonance imaging with Body Mass Index in overweight and obese adults: A population-based study. Arthritis \& Rheumatism. 2012; 64(5):1488-96. [DOI:10.1002/art.33462] [PMID]

[11] Hadjipavlou A, Tzermiadianos M, Bogduk N, Zindrick M The pathophysiology of disc degeneration: A critical review. The Journal of Bone and Joint Surgery British Volume. 2008; 90(10):1261-70. [DOI:10.1302/0301-620X.90B10.20910] [PMID]

[12] Urban JP, Roberts S. Degeneration of the intervertebral disc. Arthritis Research \& Therapy. 2003; 5(3):120-30 [DOI:10.1186/ar629] [PMCID]

[13] Letafatkar K, Hadadnezhad M, Arashpour H, Bakhsheshi Heris. [Effects of weight, gender and number of pregnancies on lumbar total and segmental lordosis and low back pain (Persian)]. Journal of Research in Rehabilitation Sciences. 2010; 4(2):137-44. [DOI:10.22122/jrrs.v4i2.46]

[14] Rahimi N, Raeisi H. [The Prevalence of low back pain and its correlation with functional disability, quality of life, and Body Mass Index in military staff (Persian)]. Sadra Medical Sciences Journal. 2017; 3(4):269-78

[15] Mashinchi S, Hojjati-Zidashti Z, Yousefzadeh-Chabok S. BMI, abdominal obesity and physical activity in adult candidates for lumbar disk degeneration surgery. Iranian Journal of Neurosurgery. 2018; 4(2):83-92. [DOI:10.32598/irjns.4.2.83]

[16] Mahan LK, Escott-Stump S. Krause's food, nutrition, \& diet therapy. Philadelphia: Saunders; 2004

[17] Takatalo J, Karppinen J, Taimela S, Niinimäki J, Laitinen J, Sequeiros RB, et al. Body Mass Index is associated with lumbar disc degeneration in young Finnish males: Subsample of Northern Finland birth cohort study 1986. BMC Musculoskeletal Disorders. 2013; 14:87. [DOI:10.1186/1471-2474-14-87] [PMID] [PMCID]

[18] Wahlström J, Burström L, Nilsson T, Järvholm B. Risk factors for hospitalization due to lumbar disc disease. Spine 2012; 37(15):1334-9. [DOI:10.1097/BRS.0b013e31824b5464] [PMID]

[19] Hadizadeh Kharazi H, Saedi D. [A study of prevalence of mri finding in patients with degenerative discovertebral low back pain (Persian)]. Razi Journal of Medical Sciences. 2002; 9(28):139-48. 
[20] BayatTorq M, Sarafraz Ardakani H, Mazidi M, Savadi M, Rafati S. [Prevalence of low back pain in school-age children and associated risk factors (Persian)]. Bimonthly Journal of Hormozgan University of Medical Sciences. 2013; 16(6):47783.

[21] Videman T, Gibbons LE, Kaprio J, Battié MC. Challenging the cumulative injury model: Positive effects of greater body mass on disc degeneration. The Spine Journal. 2010; 10(1):2631. [DOI:10.1016/j.spinee.2009.10.005] [PMID]

[22] Kauppila LI, Penttilä A, Karhunen PJ, Lalu K, Hannikainen P. Lumbar disc degeneration and atherosclerosis of the abdominal aorta. Spine. 1994; 19(8):923-9. [DOI:10.1097/00007632199404150-00010] [PMID]

[23] Nasrollahzadeh J, Sevid M, Mazlum Z. [Nutrition therapy in obesity, diabetes mellitus, and cardiovascular diseases (Persian)]. Tehran: Pelk; 2003.

[24] Adams GM. Exercise physiology laboratory [F Rahmani Nia, H Rajabi, AA Gaeni, H Mojtahedi, Persian trans.]. Tehran: Hatmi; 2014.

[25] Samartzis D, Karppinen J, Cheung JPY, Lotz J. Disk degeneration and low back pain: Are they fat-related conditions. Global Spine Journal. 2013; 3(3):133-44. [DOI:10.1055/s-0033-1350054] [PMID] [PMCID]

[26] Daneshjoo A, Dadgar H. [The prevalence of low back pain and its relationship with physical activity, age and BMI in Fars Payam-e Noor University staff (Persian)]. Journal of Research in Rehabilitation Sciences. 2011; 7(3):302-10.

[27] Pye SR, Reid DM, Adams JE, Silman AJ, O'neill TW. Influence of weight, Body Mass Index and lifestyle factors on radiographic features of lumbar disc degeneration. Annals of the Rheumatic Diseases. 2007; 66(3):426-7. [DOI:10.1136/ ard.2006.057166] [PMID] [PMCID] 